\title{
Antitumor effects of carbon nanotube-drug complex against human breast cancer cells
}

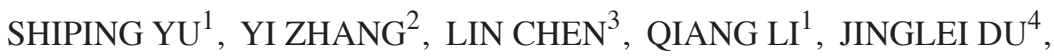 \\ YUDUAN GAO ${ }^{5}$, LI ZHANG ${ }^{6}$ and YONGZHEN YANG ${ }^{3}$
}

\author{
${ }^{1}$ Department of Interventional Therapy, The Second Hospital of Shanxi Medical University, Taiyuan, Shanxi 030001; \\ ${ }^{2}$ Department of Radiology, Peace Hospital of Changzhi Medical University, Changzhi, Shanxi 046000; ${ }^{3}$ Key Laboratory of \\ Interface Science and Engineering in Advanced Materials, Ministry of Education, Taiyuan University of Technology, \\ Taiyuan, Shanxi 030024; ${ }^{4}$ Department of Medical Imageology, Shanxi Medical University, Taiyuan, Shanxi 030001; \\ Departments of ${ }^{5}$ Ophthalmology and ${ }^{6}$ Urinary Surgery, Shanxi Dayi Hospital, Taiyuan, Shanxi 030032, P.R. China
}

Received February 4, 2017; Accepted March 27, 2018

DOI: $10.3892 /$ etm.2018.6334

\begin{abstract}
To improve the bio-solubility and sustained-release properties of a carbon nanotube (CNT)-drug complex, the present study used a hydrophilic polymer, polyethylene glycol (PEG), and $\beta$-estradiol (E2), which targets the estrogen receptor in human breast cancer cells (HBCCs), to modify CNTs carrying lobaplatin (LBP) to form E2-PEG-CNT-LBP. The in vitro inhibitory effects against HBCCs and the in vivo pharmacological effect of the complex on heart, liver and kidney tissues were also evaluated. The results indicated that the inhibitory effects of this complex against HBCCs reached $80.44 \%$ within $72 \mathrm{~h}$. A blood biochemical test of normal mice indicated that this complex reduced platelet counts, while aspartate aminotransferase levels were increased compared with those in the control group. Histopathological analysis revealed no obvious adverse effects on the heart, liver and kidneys. The in vivo results indicated that the novel E2-PEG-CNT-LBP complex had no obvious toxic effects while exhibiting sustained-release properties. The clearance of E2-PEG-CNT-LBP by non-specific uptake systems was delayed and its clearance was increased compared with LBP alone.
\end{abstract}

\section{Introduction}

Breast cancer (BC) is the most common type of malignancy in women and poses a severe health threat. The worldwide incidence of $\mathrm{BC}$ is increasing each year (1). At present, the major treatment for BC is surgical resection, which is combined with chemoradiotherapy in later stages, so as to reduce tumor

Correspondence to: Professor Shiping Yu, Department of Interventional Therapy, The Second Hospital of Shanxi Medical University, 382 Wuyi Road, Taiyuan, Shanxi 030001, P.R. China E-mail: cncspy@126.com

Key words: carbon nanotube-drug complex, lobaplatin, breast cancer cells, antitumor, target recurrence and achieve better overall therapeutic effects (2). Although traditional treatment methods are effective, they still have numerous deficiencies, e.g. cancer tissue cannot be completely resected by surgery and chemoradiotherapy has significant side effects (3). Chemotherapy is mainly based on platinum drugs, including cisplatin, carboplatin or lobaplatin (LBP). The first-generation platinum drug, cisplatin, has a limited application due to the large dosage required and its severe toxicity in to kidneys, digestive tract and nerves. The second-generation platinum drug, carboplatin, also has limited clinical efficacy and prolonged usage is required due to its wide cross-resistance with cisplatin, as well as significant inhibitory effects on the bone marrow. The third-generation platinum drug LBP has high potential for the treatment of cancer due to its high efficiency, no cross-resistance and low toxicity. Its major mechanism of action is to form cross-links with the DNA double helix in tumor cells, thus abrogating the replication function of the DNA template and further inhibiting the replication of DNA, which finally leads to the death of tumor cells. However, prolonged use of LBP may also lead to abnormalities in the blood system $(4,5)$. Therefore, research has focused on reducing the dosage of chemotherapeutic drugs, while improving the local drug concentration, as well as on the reduction of side effects (6). The development of a targeted drug with low toxicity, high efficiency and high specificity is critical for the treatment of BC.

In recent years, with the rapid development of nanodrugs, novel methods and techniques have been applied for this purpose, which have gained attention from the medical science community and materials industry (7). Nanomaterials used for the in vivo transportation of traditional drugs to improve drug targeting and utilization, while reducing toxicity, may provide a breakthrough toward the development of drugs to cure cancer and other diseases (8). Carbon nanotubes (CNTs) have attracted much attention due to their cavity-containing structure, excellent cell penetrability and relatively large specific surface area, which may be utilized to carry biologically active molecules and drugs $(9,10)$. However, as CNTs are insoluble in physiological buffer, they can produce a strong immunogenicity in the body, thus limiting their application (11). 
Polyethylene glycol (PEG) is neutral, non-toxic, non-antigenic and non-immunogenic, and has unique physicochemical properties and good biocompatibility. It may improve the bio-solubility, compatibility and drug transitivity of CNTs when applied as a coating (12). It may effectively reduce the clearance of CNTs by non-specific uptake systems in vivo (mainly in the liver and spleen) and increase the in vivo clearance of CNTs, thus indirectly increasing the duration of action against targeted tumors $(13,14)$.

Estrogen, particularly $\beta$-estradiol (E2), is essential for maintaining female reproductive development and secondary sexual characteristics. Through binding to the estrogen receptor (ER), E2 induces conformational changes and the release of molecular chaperones, including heat shock protein (HSP)90, HSP70, cyclophilin or P23; therefore, it is a necessary cofactor for the high transcription and expression of various genes in tumor cells (15-17). Compared with normal cells, cancer cells have a high expression of ER (18); therefore, targeted treatment against various hormone-sensitive tumors is also the focus of endocrine therapy toward BC $(19,20)$.

To improve the inhibitory effects on cancer cell proliferation and the anti-tumor properties of LBP, the present study selected CNTs with a high drug-loading capacity as a drug carrier to deliver LBP. First, PEG was applied to modify the surface of CNTs to increase their biocompatibility; E2 was then grafted to endow CNTs with high targeting properties. Finally, the CNT-drug complex (E2-PEG-CNT-LBP) was obtained by loading LBP onto modified CNTs. In the present study, in vitro anti-tumor effects of E2-PEG-CNT-LBP against human breast cancer cells (HBCCs) and the possible adverse effects of this targeted LBP delivery system in normal mice in vivo were investigated.

\section{Materials and methods}

Materials and animals. CNTs (purity, $99.8 \mathrm{wt} \%$; internal diameter, 5-10 nm; length, 0.8-12 $\mu \mathrm{m}$; specific surface area, $>233 \mathrm{~m}^{2} / \mathrm{g}$ ), trypsin EDTA solution, penicillin-streptomycin solution and dimethyl sulfoxide (DMSO) were purchased from Chengdu Biological Co. Ltd (Chengdu, China). PEG (average molecular weight, 2,500 Da; purity, $99.0 \mathrm{wt} \%$ ); dimethylformamide (DMF; purity, $99.5 \mathrm{wt} \%)$, chloroform $\left(\mathrm{CHCl}_{3}\right.$; purity, $99.0 \mathrm{wt} \%$ ), nitric acid (purity, $68.0 \mathrm{wt} \%$ ) and concentrated sulfuric acid (purity, $98.0 \mathrm{wt} \%$ ) were obtained from Sinopharm Chemical Reagent Co. Ltd, (Shanghai, China). E2 (purity, $99.0 \mathrm{wt} \%$ ), Propidium Iodide Staining Kit (with 10X Buffer A), fetal bovine serum, RPMI 1640 medium and Dulbecco's modified Eagle's medium (DMEM)/high glucose (1X) were purchased from Boke Biobase Co. Ltd (Jinan, China). LBP (purity, $95.0 \mathrm{wt} \%$ ) was purchased from Chang'an International Pharm Co. Ltd (Haikou, China). The HBCC cell line MCF-7 and fetal bovine serum were purchased from Goybiotech Co. Ltd (Shanghai, China). MTT was purchased from Sigma-Aldrich (Merck KGaA, Darmstadt, Germany). A total of 42 healthy female C57BL/6 mice (age, 4-6 weeks; weight, 18-20 g) were provided by Beijing Vital River Laboratory Animal Technology Co., Ltd (Beijing, China).

Preparation of E2-PEG-CNT-LBP. CNTs were first bound to PEG so as to increase the dispersion and loading rate of the
CNTs in the solvent. The complex was then combined with E2, followed by LBP, in order to obtain the final complex, E2-PEG-CNT-LBP.

PEG modification of CNT. First, the appropriate amount of CNTs was reacted with concentrated nitric acid for $24 \mathrm{~h}$ under ultrasonic conditions, and then reacted with a 3:1 mixture of concentrated nitric acid and concentrated sulfuric acid for $4 \mathrm{~h}$. After the reaction, a black precipitate was observed after the mixture was allowed to stand still. Following removal of the supernatant, the black precipitate was washed using triple distilled water, and dried for $8 \mathrm{~h}$ in an oven at $75^{\circ} \mathrm{C}$. CNTs carrying- $\mathrm{COOH}$ moieties (CNT-COOH) were then obtained.

Subsequently, $300 \mathrm{mg}$ of CNT-COOH was refluxed with $20 \mathrm{ml}$ of DMF and 5 drop of thionyl chloride $\left(75^{\circ} \mathrm{C}\right)$ for $12 \mathrm{~h}$, cooled to room temperature, and then dried at $100^{\circ} \mathrm{C}$ in a vacuum to obtain the CNTs. Next, the hydrophilic PEG was loaded onto the chlorinated CNTs. The mixture was then reacted with $15 \mathrm{ml}$ of $\mathrm{CHCl}_{3}, 0.1 \mathrm{ml}$ of PEG and 5 drop of DMF for $10 \mathrm{~h}$, washed 3 times with ethanol ( $5 \mathrm{~min} /$ wash), and dried for $8 \mathrm{~h}$ in an oven at $75^{\circ} \mathrm{C}$ to obtain PEG-coated CNTs (PEG-CNT).

Preparation of E2-PEG-CNT. First, $5 \mathrm{mg}$ E2 dissolved in $0.5 \mathrm{ml}$ absolute ethanol was ultrasonicated in an ice bath for 30 min to fully dissolve E2, and this solution was then added to $5 \mathrm{mg}$ PEG-CNTs. This mixture was ultrasonicated in an ice bath for 15 min to fully mix E2 and PEG-CNTs. Subsequently, an appropriate amount of triple-distilled water was slowly added to the mixture with ultrasonication, followed by ultrasonic crushing (10 times at $400 \mathrm{~W}, 30 \mathrm{sec}$ each time) in an ice bath. The supernatant obtained after 20 min of centrifugation at $42,931.2 \times \mathrm{g}$ and $0-4^{\circ} \mathrm{C}$ was removed, and the black residue was subjected to the above procedures two more times in order to remove the remaining solvent and obtain E2-PEG-CNT.

Preparation of E2-PEG-CNT-LBP. The procedure of loading LBP onto the surface of the E2-PEG-CNT was performed according to a previous study (21). The specific processes were as follows: PEG-CNT (or E2-PEG-CNT) and LBP (mass ratio, 2:1) were added to PBS $(\mathrm{pH}=7.4)$. The mixture was kept in an incubator maintained at $37^{\circ} \mathrm{C}$ for $24 \mathrm{~h}$ and then centrifuged. The ultraviolet-visible light (UV-vis) absorption spectrum of the supernatant was then measured. The drug-loading rate was calculated as follows:

Drug loading rate $(\%)=(\mathrm{A} 1-\mathrm{A} 2) / \mathrm{A} 1 \times 100 \%$

Entrapment rate $\%=(\mathrm{m} 2 / \mathrm{m} 1) \times 100 \%$

where $\mathrm{A} 1$ is the absorbance of LBP, A2 is the absorbance of the supernatant after centrifugation, $\mathrm{m} 2$ is the mass of the carrier after drug loading [ $\mathrm{m} 2=\mathrm{m} 1 \times$ x (1+ drug loading rate) $]$ and $\mathrm{m} 1$ is the mass of the carrier prior to drug loading. The obtained drug-loaded complexes were labeled as PEG-CNT-LBP and E2-PEG-CNT-LBP as appropriate.

Cell culture. The HBCC cell line MCF-7 was cultured in RPMI 1640 medium supplemented with $10 \%$ fetal bovine serum and $1 \%$ penicillin-streptomycin at $37^{\circ} \mathrm{C}$ in a humidified 
atmosphere with $5 \% \mathrm{CO}_{2}$. Cells in the logarithmic phase were used for the experiments.

In vitro tumor cell inhibition experiment. The inhibitory rates of LBP, CNT-LBP, PEG-CNT, E2-PEG-CNT and E2-PEG-CNT-LBP against the MCF-7 HBCC cell line were detected with an MTT assay. First, the reagent solutions were prepared at concentrations of $0,50,100$, and $200 \mu \mathrm{g} / \mathrm{ml}$ in RPMI 1640 medium; the preparations were autoclaved at $121^{\circ} \mathrm{C}$ for $30 \mathrm{~min}$ and sealed for future use. The samples were dispersed by ultrasound for $30 \mathrm{~min}$ prior to use. The concentration of MTT in the MTT assay was $5 \mathrm{mg} / \mathrm{ml}$, which was prepared using 10x Buffer A and by 20 -fold dilution with ultra-pure water.

Following digestion with trypsin, the cells were counted and a cell suspension $\left(1 \times 10^{5}\right.$ cells $\left./ \mathrm{ml}\right)$ was prepared, which was inoculated into 96-well culture plates (100 $\mu \mathrm{l} /$ well) overnight. Drug solutions were added to achieve final concentrations of 50,100 or $200 \mu \mathrm{g} / \mathrm{ml}$, while the control group was treated with RPMI 1640 medium only, and each condition was set up in 5 wells. The cells were incubated at $37^{\circ} \mathrm{C}$ with $5 \% \mathrm{CO}_{2}$ for 24 , 48 or $72 \mathrm{~h}$. MTT working solution $(20 \mu \mathrm{l})$ was then added, followed by further incubation for $5 \mathrm{~h}$. Following the removal of the supernatant, $150 \mu \mathrm{l}$ of DMSO was added and the plates were agitated until the formazan crystals were fully dissolved. The inhibitory rate of each complex/drug on MCF-7 cells was calculated by determining the optical density (OD) at $560 \mathrm{~nm}$ as follows:

Inhibitory rate $(\%)=[1-(\mathrm{OD}$ value of the experimental group-OD value of the blank control group)/(OD value of the control group-OD value of the blank control group)] x $100 \%$.

In vivo evaluation. The present study then assessed whether the targeted drug delivery system exhibits reduced toxicity compared with that of the drug itself in normal mice. For this, routine blood parameters and biochemical indexes were assessed, and the heart, liver and kidney tissues were histologically examined.

A total of 42 healthy female mice were randomly divided into 7 groups, namely the CNT, PEG-CNT, E2-PEG-CNT, PEG-CNT-LBP, E2-PEG-CNT-LBP, LBP and normal saline control (NS) groups $(n=6)$, and were administered the corresponding agents on the 1st and 7th day by injection through the tail vein. The concentration of LBP was $0.5 \mathrm{mg} / \mathrm{ml}$, and the total dose was $5 \mathrm{mg} / \mathrm{kg}$ over the course of the experiment. PEG-CNT and E2-PEG-CNT were used as LBP carriers. The entrapment rates were 115 and $120 \%$, respectively. To ensure that the LBP concentration in the CNT complex suspension was $0.5 \mathrm{mg} / \mathrm{ml}$, the total concentration of PEG-CNT-LBP and E2-PEG-CNT-LBP was adjusted to 0.87 and $0.83 \mathrm{mg} / \mathrm{ml}$, respectively, so that the $\mathrm{LBP}$ concentration could be maintained at $5 \mathrm{mg} / \mathrm{kg}$ (22). In the PEG-CNT-LBP and E2-PEG-CNT-LBP groups, the concentration of PEG-CNT and E2-PEG-CNT was 0.3 and $0.25 \mathrm{mg} / \mathrm{ml}$, respectively, and in the CNT and LBP groups, the drug concentration was $0.5 \mathrm{mg} / \mathrm{ml}$; the control group was injected with the same volume of NS (22).

In order to avoid overdosing, the injected volume of drug solution injected did not exceed $0.6 \mathrm{ml}$. Therefore, the concentration of LBP was $0.5 \mathrm{mg} / \mathrm{ml}$ and the dose for each mouse was $30 \mathrm{mg} / \mathrm{kg}$. According to the loading rates of
PEG-CNT and E2-PEG-CNT as the drug carriers, the drug concentrations in the mice were required to be the identical to that of pure LBP; the drug concentrations were thus appropriately adjusted.

On day 14, blood was sampled from the eye orbit of each mouse, followed by the use of EOTA for anticoagulation for the blood routine assay, as well as for determining alanine aminotransferase (ALT), aspartate aminotransferase (AST) and creatinine $(\mathrm{Cr})$ levels. The mice were then euthanized, and the heart, liver and kidneys were sampled for histopathological examination, by fixing with $10 \%$ formalin, embedding in paraffin, slicing and staining with $\mathrm{HE}$ in order to observe the changes in the cells and tissue structures.

Statistical analysis. The results were statistically analyzed using SPSS 18.0 (SPSS, Inc., Chicago, IL, USA). Values are expressed as the mean \pm standard deviation, and the average values of multiple samples were analyzed by single-factor analysis of variance followed by a Dunn's Multiple Comparison test. $\mathrm{P}<0.05$ was considered to indicate a statistically significant difference. The number of degrees of freedom (Df) was calculated, and F-statistics were performed to assess heterogeneity.

\section{Results}

Water solubility. Fig. 1 presents images of CNTs prior to and after PEG modification dispersed in deionized water for $48 \mathrm{~h}$. The images indicate that unmodified CNTs agglomerated in water and formed a precipitate. The water solubility of CNT-COOH was better than that of CNT, and although a small amount of precipitate formed, the dispersion was significantly improved. In addition, the dispersibility of CNTs grafted by PEG was improved. Due to the grafting of PEG on the surface of CNT, it was able to form effective electrostatic interactions, which inhibit the aggregation of CNT.

$U V$-vis spectra. The UV-vis spectra of PEG-CNT, E2-PEG-CNT and E2-PEG-CNT-LBP are presented in Fig. 2. The UV-vis spectra of PEG-CNT and of E2-PEG-CNT prior to and after LBP loading were obtained by scanning within the UV-vis wavelength range. The absorption peak of the CNT group of PEG-CNT was at $250 \mathrm{~nm}$, while E2-PEG-CNT exhibited a peak for E2 absorption at $\sim 200 \mathrm{~nm}$ and a peak for $\mathrm{CNT}$ at $265 \mathrm{~nm}$; after loading with LBP, the absorption peak of CNT shifted to $280 \mathrm{~nm}$ in E2-PEG-CNT-LBP.

Inhibitory effects. In Fig. 3, the MCF-7 cell inhibitory rates are compared among the different compounds at various concentrations and incubation times. The growth inhibitory rate of MCF-7 cells in the different groups increased with the prolongation of the interaction between the cells with LBP or the drug-loaded complex, compared with that in the control group. The inhibitory rate in the $200 \mu \mathrm{g} / \mathrm{ml}$ E2-PEG-CNT-LBP group increased from 19.72 to $80.44 \%$ when the incubation time was markedly increased from 24 to $72 \mathrm{~h}$. Furthermore, for the same treatment duration, the inhibitory rate increased with the drug concentration, and this difference was marked when compared with the control group. When MCF-7 cells were treated with the drug-loaded complex for $24 \mathrm{~h}$, the inhibition rate increased 


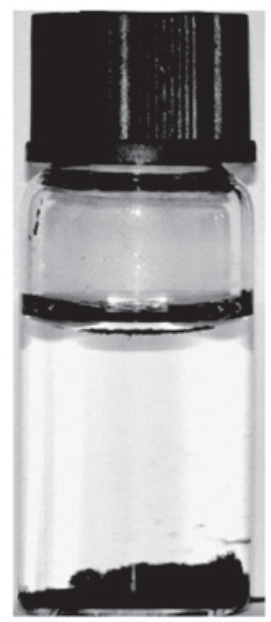

CNT

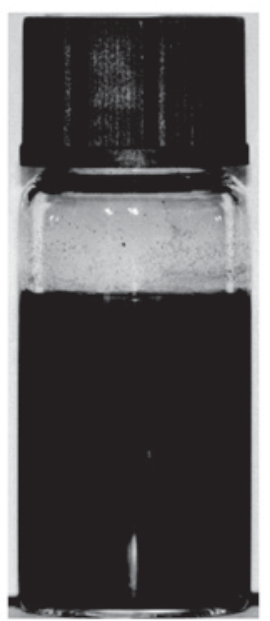

$\mathrm{CNT}-\mathrm{COOH}$

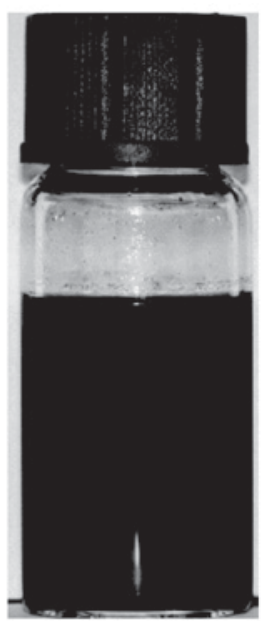

PEG-CNT
Figure 1. Images of CNT, CNT-COOH and PEG-CNT dispersed in deionized water for $48 \mathrm{~h}$. CNT, carbon nanotubes; PEG, polyethyleneglycol.

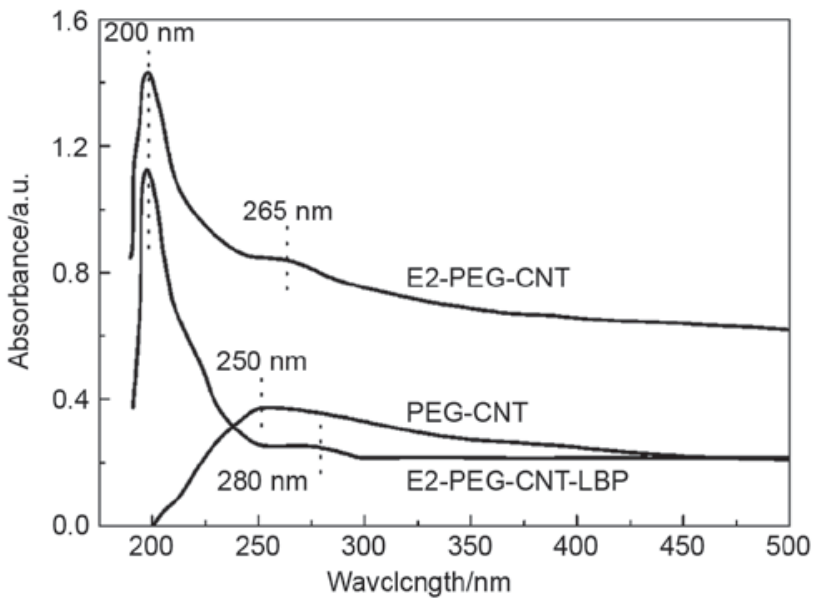

Figure 2. Ultraviolet-visible light adsorption spectra of PEG-CNT, E2-PEG-CNT and E2-PEG-CNT-LBP. CNT, carbon nanotubes; PEG, polyethyleneglycol; E2, $\beta$-estradiol; LBP, lobaplatin; a.u., absorption units.

from 7.71 to $19.72 \%$ when the concentration of the complex was increased from 50 to $200 \mu \mathrm{g} / \mathrm{ml}$.

At each time point, the inhibition rates in the LBP and E2-PEG-CNT-LBP groups were significantly greater compared with the control group. At $72 \mathrm{~h}$, the inhibition rates in the E2-PEG-CNT-LBP groups were significantly greater compared with the LBP. The inhibitory rate in the $200 \mu \mathrm{g} / \mathrm{ml}$ E2-PEG-CNT group were significantly greater compared with the control group at each time point. The inhibitory rate in the 100 and $200 \mu \mathrm{g} / \mathrm{ml}$ PEG-CNT group were significantly greater compared with the control group at 48 and $72 \mathrm{~h}$. The inhibition rates in the PEG-CNT groups were significantly greater compared with the control group at $72 \mathrm{~h}$. At each time point, the inhibition rates in the PEG-CNT and E2-PEG-CNT groups were significantly lower compared with the LBP group.

The morphology of normal MCF-7 cells (Fig. 4A) and that after culture with E2-PEG-CNT-LBP for 48 h (Fig. 4B) was observed using an inverted microscope (magnification, $\mathrm{x} 100$ ). While the untreated MCF-7 cells exhibited good adherence, had a shuttle-like or polygonal shape and had high transparency, most of the cells died following prolonged treatment with the drug-loaded complex.

Blood routine and biochemical indexes. The present study investigated the possible adverse effects of E2-PEG-CNT-LBP in vivo, and the LBP dosage in each group of mice was set at $5 \mathrm{mg} / \mathrm{kg}$. Each group was injected the respective drug on D1 and D7 through the tail vein; in order to avoid causing excessive reactions in mice, the amount did not exceed $0.6 \mathrm{ml}$, dose of the drug was $30 \mathrm{mg} / \mathrm{kg}$ and the injection volume was $0.01 \mathrm{ml} / \mathrm{g}$. According to the entrapment rates of PEG-CNT and E2-PEG-CNT, and in order to ensure that the LBP concentration released from the complex was equal to that of pure LBP, their concentrations were appropriately adjusted.

Blood biochemical parameters, including blood routine values, and parameters of liver and renal function, were compared among the groups in order to determine whether the drugs caused any abnormalities in organ function in the early stages (Table I).

As presented in Table I, it was identified that, compared with the control group, unmodified CNT, PEG-CNT and E2-PEG-CNT did not cause any abnormality of the WBC and $\mathrm{Hb}$. Compared with the PLT count in the control group, that in the E2-PEG-CNT-LBP, PEG-CNT-LBP and LBP groups was significantly lower; furthermore, the PLT count in the LBP group was significantly lower than that in the PEG-CNT-LBP and E2-PEG-CNT-LBP groups. The level of AST in the CNT group was significantly higher than that in the control group.

The differences in Hb, WBC, PLT, ALT, AST and Cr levels in the C57BL/6 mice of each group were examined. The results on PLT, ALT, AST and Cr exhibited a significant difference compared with the control group.

Compared with the control group, the level of PLT, ALT and AST in the CNT group was significantly higher, and CR was significantly higher and lower in the PEG-CNT and E2-PEG-CNT group, respectively. Compared with the CNT group, the level of PLT, ALT, and AST was significantly lower in the PEG-CNT and E2-PEG-CNT group, and CR was significantly higher and lower in the PEG-CNT and E2-PEG-CNT groups, respectively. Compared with the PEG-CNT group, the level of AST in the E2-PEG-CNT group was significantly higher. Compared with the LBP group, the level of PLT and ALT was significantly higher and CR was significantly lower in the PEG-CNT-LBP and E2-PEG-CNT-LBP groups. The level of AST was significantly higher in the LBP group and significantly lower in the PEG-CNT-LBP group compared with the E2-PEG-CNT-LBP group. Compared with the PEG-CNT-LBP group, the level of PLT and ALT in the E2-PEG-CNT-LBP group was significantly lower.

Histopathology. Fig. 5A displays histopathological images of the hearts of mice injected with the CNT-drug complexes. The morphology of the myocardium was regular, the nucleus exhibited integrity. The arrangement of myofibers was normal and the structure was clear, without any oozing, hyperemia and edema. In addition, no indication of cardiomyocyte degeneration was observed in any group.

Fig. 5B presents histopathological images of the kidneys. No obvious renal tubular epithelial cell atrophy or vacuolar 
A

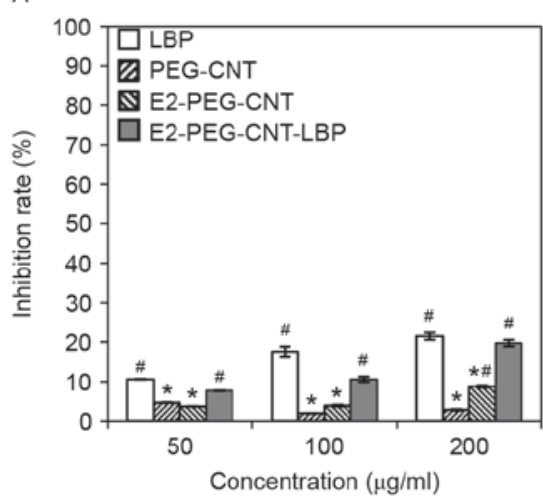

B

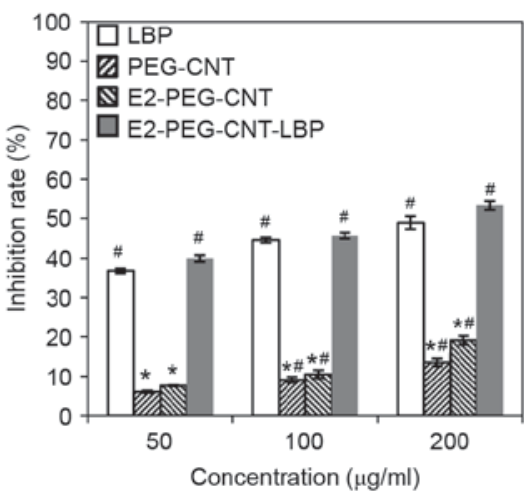

C

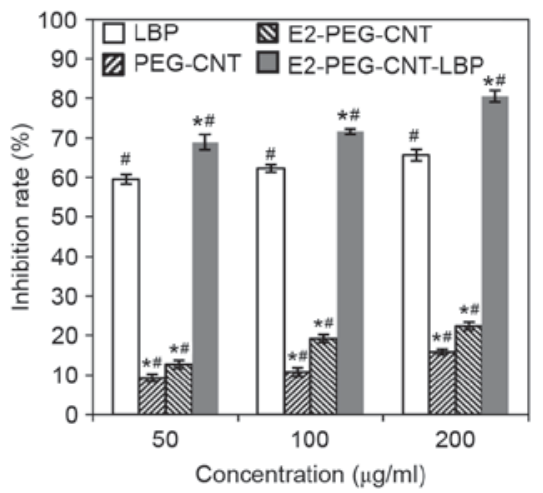

Figure 3. Inhibitory rates of LBP, PEG-CNT, E2-PEG-CNT and E2-PEG-CNT-LBP against MCF-7 at different concentrations after incubation for (A) $24 \mathrm{~h}$, (B) $48 \mathrm{~h}$ and (C) $72 \mathrm{~h}$. The inhibition rate of the control group was set at $0 \% .{ }^{\prime \prime} \mathrm{P}<0.01 \mathrm{vs}$. the control group; ${ }^{*} \mathrm{P}<0.01 \mathrm{vs}$. the LBP group. CNT, carbon nanotubes; PEG, polyethyleneglycol; E2, $\beta$-estradiol; LBP, lobaplatin.

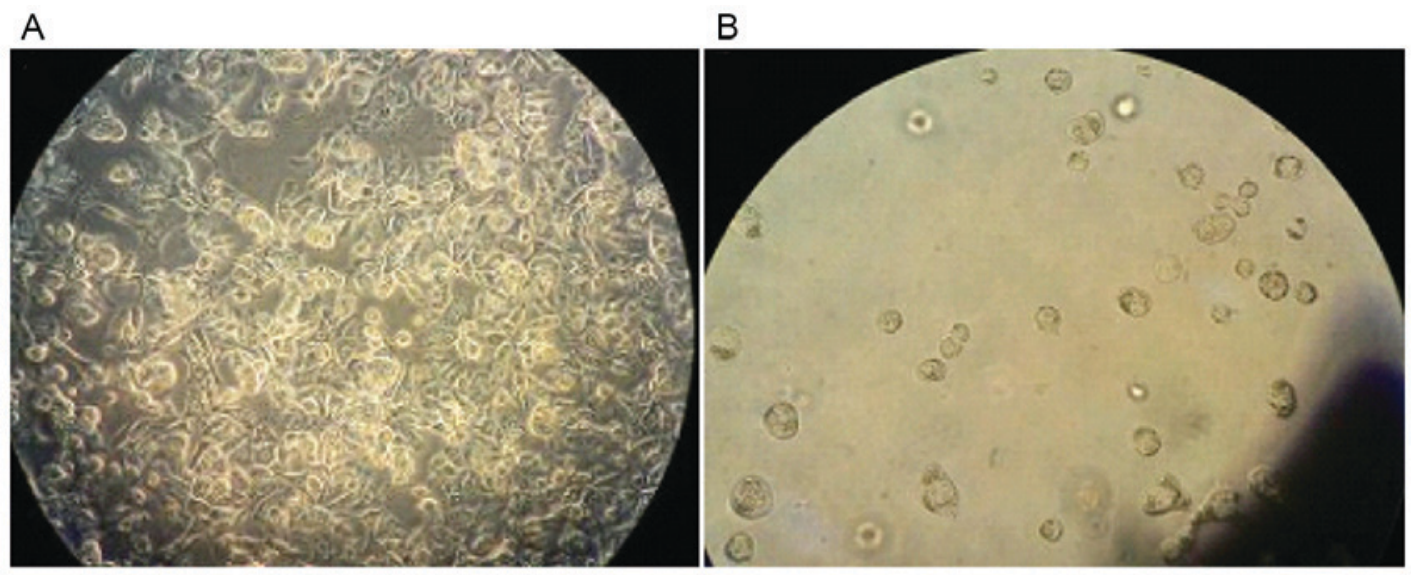

Figure 4. Morphology of (A) normal MCF-7 cells and (B) those after culture with $50 \mu \mathrm{g} / \mathrm{ml}$ E2-PEG-CNT-LBP for $48 \mathrm{~h}$ observed under an inverted microscope (magnification, x100). CNT, carbon nanotubes; PEG, polyethyleneglycol; E2, $\beta$-estradiol; LBP, lobaplatin.

changes were present, and no protein, uric acid crystallization or congestion was observed in the tubules in any experimental group. No interstitial inflammation was present and the structure was normal compared with that in the blank control group.

In Fig. 5C, histopathological images of the liver are displayed. The liver cells exhibited a cord-like radial pattern arrangement, with intact morphological structure, a clear central vein, no necrosis of liver cells or abnormal pathological changes. Compared with the NS group, no peripheral inflammatory exudation was identified in the liver portal area and hepatic portal tissue structure in any experimental group.

\section{Discussion}

The improved dispersibility of CNTs by PEG may be due to the grafting of PEG onto the CNTs, resulting in the formation of an effective electrostatic layer in solution. This anti-static force among the CNTs overcomes gravity and van der Waals forces, so that the water solubility is enhanced. This result confirms that the modification of the surface of the CNTs by PEG improves the biocompatibility of the CNTs.

The UV-vis spectrum of E2-PEG-CNT exhibited the characteristic absorption peaks of E2 near $200 \mathrm{~nm}$ and CNT at $265 \mathrm{~nm}$ (23), indicating that E2 was loaded onto the PEG-CNT surface. Compared with the UV-vis curve of E2-PEG-CNT, the absorption peak of E2-PEG-CNT-LBP at $265 \mathrm{~nm}$ shifted to $\sim 280 \mathrm{~nm}$, which may be due to the introduction of LBP, confirming that LBP was adsorbed onto the surface of E2-PEG-CNTs.

The inhibitory rate of LBP on MCF-7 cells relative to the control group increased in a time-dependent manner. At the same time-point, the inhibitory rate increased with the increase in drug concentration in a dose-dependent manner. Therefore, it may be concluded that LBP has a significant time- and dose-dependent inhibitory effect on BC cells. By contrast, PEG-CNT and E2-PEG-CNT had less pronounced inhibitory effects on the proliferation of $\mathrm{HBCCs}$ at any concentration, which may be due to the toxicity of CNTs being decreased due to PEG modification. Of note, E2 is known to affect HBCCs including MCF-7 cells as a ligand, which may promote their proliferation, but this was apparently counteracted by the low toxicity of CNT-PEG. By contrast, LBP and E2-PEG-CNT-LBP significantly inhibited the proliferation of HBCCs $(\mathrm{P}<0.05)$. This is due to the known cytotoxic and growth inhibitory effects of LBP, including inhibition of cell cycle progression, and the results further confirmed that LBP was successfully loaded onto the surface of E2-PEG-CNT. Comparison of the inhibitory rates on HBCCs between LBP and E2-PEG-CNT-LBP reveals that the inhibitory rate of 
Table I. Results of blood biochemical indexes in different groups.

\begin{tabular}{lcccccc}
\hline Group & Hb $(\mathrm{g} / \mathrm{l})$ & WBC $\left(10^{9} / \mathrm{l}\right)$ & PLT $\left(10^{9} / \mathrm{l}\right)$ & ALT $(\mathrm{U} / \mathrm{l})$ & AST $(\mathrm{U} / \mathrm{l})$ & CR $(\mu \mathrm{mol} / \mathrm{l})$ \\
\hline CNT & $134.39 \pm 6.05$ & $6.29 \pm 1.20$ & $360.93 \pm 87.60^{\mathrm{a}}$ & $66.16 \pm 5.81^{\mathrm{a}}$ & $165.66 \pm 4.67^{\mathrm{a}}$ & $56.16 \pm 3.48$ \\
PEG-CNT & $138.09 \pm 3.68$ & $6.83 \pm 1.19$ & $320.00 \pm 71.22^{\mathrm{b}}$ & $48.50 \pm 6.15^{\mathrm{b}}$ & $124.00 \pm 15.08^{\mathrm{b}}$ & $62.00 \pm 3.03^{\mathrm{a}, \mathrm{b}}$ \\
E2-PEG-CNT & $136.02 \pm 7.38$ & $5.99 \pm 1.21$ & $337.50 \pm 106.3^{\mathrm{b}}$ & $48.11 \pm 2.66^{\mathrm{b}}$ & $139.00 \pm 9.67^{\mathrm{b}, \mathrm{c}}$ & $53.66 \pm 3.07^{\mathrm{a}-\mathrm{c}}$ \\
PEG-CNT-LBP & $136.58 \pm 5.20$ & $6.83 \pm 1.64$ & $123.13 \pm 43.91^{\mathrm{a}-\mathrm{d}}$ & $65.96 \pm 9.94^{\mathrm{a}, \mathrm{c}, \mathrm{d}}$ & $123.50 \pm 8.80^{\mathrm{a}, \mathrm{b}, \mathrm{d}}$ & $56.66 \pm 8.33^{\mathrm{c}, \mathrm{d}}$ \\
E2-PEG-CNT-LBP & $137.62 \pm 6.87$ & $6.21 \pm 1.32$ & $101.66 \pm 18.02^{\mathrm{a}-\mathrm{e}}$ & $54.00 \pm 8.00^{\mathrm{a}-\mathrm{e}}$ & $134.00 \pm 14.68^{\mathrm{a}-\mathrm{e}}$ & $58.16 \pm 4.44^{\mathrm{c}, \mathrm{d}}$ \\
LBP & $136.42 \pm 8.26$ & $7.11 \pm 1.64$ & $54.00 \pm 12.13^{\mathrm{a}-\mathrm{f}}$ & $65.65 \pm 7.41^{\mathrm{a}, \mathrm{c}, \mathrm{d}, \mathrm{f}}$ & $117.50 \pm 10.13^{\mathrm{a}-\mathrm{f}}$ & $63.16 \pm 7.33^{\mathrm{a}, \mathrm{b}, \mathrm{d}, \mathrm{e}, \mathrm{f}}$ \\
NS & $138.28 \pm 3.78$ & $6.53 \pm 1.14$ & $328.16 \pm 59.11$ & $49.80 \pm 3.54$ & $130.04 \pm 20.64$ & $57.66 \pm 6.49$
\end{tabular}

Normal range for each biochemical index is described in Wang et al $(25)$. ${ }^{\mathrm{a}} \mathrm{P}<0.01 \mathrm{vs}$. the NS group; ${ }^{\mathrm{b}} \mathrm{P}<0.01 \mathrm{vs}$. the $\mathrm{CNT}$ group; ${ }^{\mathrm{P}}<0.01 \mathrm{vs}$. the PEG-CNT group; ${ }^{d} \mathrm{P}<0.01$ vs. the E2-PEG-CNTgroup; ${ }^{\mathrm{e}} \mathrm{P}<0.01$ vs. The PEG-CNT-LBP group; ${ }^{\mathrm{P}}<0.01$ vs. the PEG-CNT-LBP group. $\mathrm{Hb}$, hemoglobin; WBC, white blood cells; PLT, platelets; ALT, alanine aminotransferase; CNT, carbon nanotubes; PEG, polyethyleneglycol; E2, $\beta$-estradiol; LBP, lobaplatin; NS, normal saline.

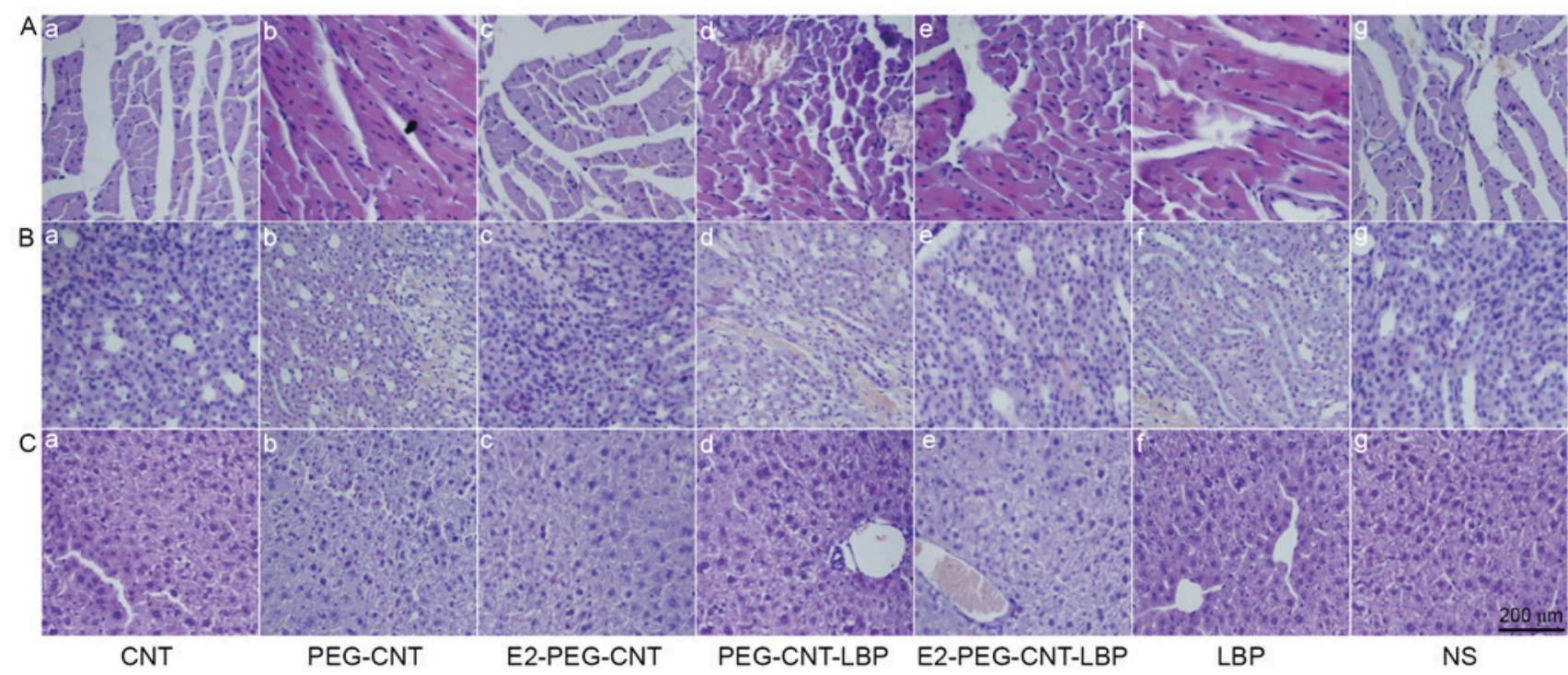

Figure 5. Representative histopathological images of (A) heart, (B) kidney and (C) liver tissues in groups treated with (a) CNT, (b) PEG-CNT, (c) E2-PEG-CNT, (d) PEG-CNT-LBP, (e) E2-PEG-CNT-LBP, (f) LBP and (g) NS (scale bar, $200 \mu \mathrm{m}$ ). CNT, carbon nanotubes; PEG, polyethyleneglycol; E2, $\beta$-estradiol; LBP, lobaplatin; NS, normal saline.

E2-PEG-CNT-LBP is significantly higher than that of pure LBP when the incubation time is $72 \mathrm{~h}$. This may be the result of two responses: i) E2 can target ER in $\mathrm{MCF}-7$, which increases the inhibitory effect of LBP against the HBCCs; ii) PEG-modified CNTs may have an increased clearance, thus indirectly increasing the target acting time of LBP toward the $\mathrm{BC}$ cells. With the increased incubation time and specific targeting of $\mathrm{BC}$ cells, the drug dosage and adverse reactions were reduced, so that satisfactory results were obtained. The apoptotic effects of E2-PEG-CNT-LBP against MCF-7 at $72 \mathrm{~h}$ were further confirmed by microscopy observation.

The possible adverse effects of the drugs were then assessed in an in vivo experiment in mice, in which blood routine and blood biochemical parameters were measured. The results indicated that the CNT, PEG-CNT and E2-PEG-CNT groups did not cause any abnormalities in the $\mathrm{WBC}$ and $\mathrm{Hb}$, when compared with the control group. This may be due to the reason that the concentrations in each experimental group did not reach toxic levels, which may have caused abnormalities in the blood system, liver and renal function. In addition to this, mice have a faster metabolism, which may lead to faster excretion of the CNTs and the drug. Therefore, no abnormality in the above parameters appeared. Compared with the PLT count in the control group, that in the E2-PEG-CNT-LBP, PEG-CNT-LBP and LBP group was significantly decreased, and the PLT count in the LBP group was significantly lower than that in the other two groups. These results indicated that the LBP-containing drugs decrease the PLT count, suggesting that the major side effect of LBP is the decrease in PLT count. However, compared with the effect of pure LBP on the PLT count, the decrease in the PLT count in the PEG-CNT-LBP and E2-PEG-CNT-LBP groups was smaller, which suggested that administration of LBP as a CNT complex reduces the adverse effect on the PLT count. This may be attributable to the fact that PEG is neutral, non-toxic, non-antigenic and non-immunogenic, has unique physicochemical properties and exhibits good biocompatibility. Therefore, when it is 
used as a functional group to modify CNTs, the complex slowly releases LBP, which reduces the dose of free LBP in the body decreases the impact on the body. Compared with the AST levels in the control group, those in the CNT group were significantly increased, consistent with previously reported results (24), indicating that CNT accumulates in the liver through the blood circulation, thus causing oxidative stress reactions in partial hepatocytes and increasing the level of AST. The results indicated that the PEG-modified CNTs effectively reduce the clearance of CNT in vivo by a nonspecific uptake system (mainly the liver and spleen) and increase the retention time in the blood circulation system, thus indirectly increasing the action time toward the targeted tumor (24).

In the present study, histopathological observation of the heart, liver and kidney indicated that the toxicity of PEG-CNT is low, and that it is therefore suitable as a targeting drug carrier. Furthermore, LBP had no obvious adverse effects on the heart, liver and kidneys, and mainly affected the blood system.

In order to further validate the cancer cell inhibition rate of E2-PEG-CNT-LBP and facilitate its examination in preclinical studies, the optimized CNT drug-loaded complex will be used in a clonogenic assay. Furthermore, signaling pathways associated with the inhibition of MCF-7 cells will be assessed and apoptosis will be examined. This will provide a broader basis for the optimization of the material structures and properties. Furthermore, the inhibition of BC by E2-PEG-CNT-LBP will be investigated in tumor-bearing mice, which will lay a solid foundation for its in vivo application.

In conclusion, in order to enhance the targeted anti-tumor therapeutic effects of LBP, the present study coated CNTs with PEG and loaded them with E2 and LBP to ultimately achieve a novel CNT-drug complex, namely E2-PEG-CNT-LBP. Furthermore, the in vitro antitumor effects of this drug-loaded complex and in vivo effects of this drug-loaded complex in the blood and tissues were also investigated. The conclusions are as follows: i) The E2-PEG-CNT-LBP drug-loaded complex has higher time- and dose-dependent cytotoxic effects on MCF-7 cells than LBP alone and ii) blood routine, liver function, and renal function tests in normal mice revealed no significant differences in the levels of $\mathrm{Hb}$ and $\mathrm{WBC}$ count among different groups.

The groups administered with LBP-containing drugs also had reduced PLT counts, indicating that the major side effect of LBP is the reductions of the PLT count. However, compared with the effect of pure LBP on the PLT count, the decrease in the PLT count in the E2-PEG-CNT-LBP group was smaller, indicating that due to PEG-modified CNT achieving a slow release of LBP, the impact of LBP on the PLT count is reduced. In addition, the results from the current study and a previous study (24) demonstrate that administration of the CNTs increased the AST and ALT levels compared with those in the control group, while, after modification with PEG, the clearance of CNT was reduced by an in vivo nonspecific uptake system. The CR levels were statistically significant, but the changes in each group were small; therefore the clinical significance was small, considering the time of observation and the dosage.

Simultaneously, it increases the retention time of the drug in the blood circulation, thereby indirectly increasing the action time on the target tumor. Histopathological analysis demonstrated that the liver tissue exhibited no significant hepatocyte necrosis or inflammatory exudation around the portal vein, cardiac histopathology indicated no myocardial change, and renal histopathology revealed no significant tubular necrosis. This indicated that the platinum drugs have no significant adverse effect on the heart, liver and kidneys, while only having a certain impact on the blood system; at the same time, small doses of CNT had no toxic effect.

In summary, the CNT drug-loaded complex has potent time- and dose-dependent inhibitory effects on MCF-7 cells. In normal mice, the adverse effects of the complex were smaller than those of pure LBP. The present study provides a theoretical and practical basis for effective targeted therapy with CNTs as a drug carrier.

\section{Acknowledgements}

Not applicable.

Funding

No funding was received.

\section{Availability of data and materials}

All data are available from the corresponding author on reasonable request.

\section{Authors' contributions}

SY was the major designer of this work and he designed the whole scheme of the experiment. YZ performed the inhibitory experiments of the nanomaterials on the MCF-7 cells. LC prepared and characterized the carbon nanotubes and modified carbon nanotubes. QL evaluated the toxicity of the nanomaterials in normal mice by investigated their blood parameters and biochemical indexes. JD statistically analyzed the results of inhibitory experiments. YG histologically examined the heart, liver and kidney tissues of mice. $\mathrm{LZ}$ wrote the scheme of the toxicity evaluation in vivo, interpreted the evaluation data and drafted the manuscript. YY involved in proposing the synthesis method of the modified carbon nanotubes, analyzing the characterization results, drafting the manuscript and revising it critically for important intellectual content. All authors read and approved the final manuscript.

\section{Ethical approval and consent to participate}

The animal study protocols were approved by the Ethics Committee of Shanxi Medical University (Taiyuan, China).

\section{Patient onsent for publication}

Not applicable.

\section{Competing interests}

The authors declare no competing interests. 


\section{References}

1. Grantzau T and Overgaard J: Risk of second non-breast cancer after radiotherapy for breast cancer: A systematic review and meta-analysis of 762,468 patients. Radiother Oncol 114: 56-65, 2015.

2. Bartelink H, Maingon P, Poortmans P, Weltens C, Fourquet A, Jager J, Schinagl D, Oei B, Rodenhuis C, Horiot JC, et al: Whole-breast irradiation with or without a boost for patients treated with breast-conserving surgery for early breast cancer: 20 -year follow-up of a randomised phase 3 trial. Lancet Oncol 16: 47-56, 2015.

3. Giordano SH: Breast cancer chemotherapy cost variation substantial in the US. Pharmacoeconomics Outcomes News 764 9-9, 2016.

4. Bhatt S, Valamanesh F, Pulpytel J, Lo Dico R, Baiyukha A, Al-Dybiat I, Pocard M, Arefi-Khonsari F and Mirshahi M: Radio-frequency plasma polymerized biodegradable carrier for in vivo release of cis-platinum. Oncotarget 7: 58121-58132, 2016

5. Guan X, Ma F, Fan Y, Zhu W, Hong R and Xu B: Platinum-based chemotherapy in triple-negative breast cancer: A systematic review and meta-analysis of randomized-controlled trials Anticancer Drugs 26: 894-901, 2015.

6. Laiva AL, Venugopal JR, Karuppuswamy P, Navaneethan B, Gora A and Ramakrishna S: Controlled release of titanocene into the hybrid nanofibrous scaffolds to prevent the proliferation of breast cancer cells. Int J Pharm 483: 115-123, 2015.

7. Beck-Broichsitter M, Merkel OM and Kissel T: Controlled pulmonary drug and gene delivery using polymeric nano-carriers. J Control Release 161: 214-224, 2012.

8. Kim JY, Choi WI, Kim YH and Tae G: Brain-targeted delivery of protein using chitosan- and RVG peptide-conjugated, pluronic-based nano-carrier. Biomaterials 34: 1170-1178, 2013.

9. Dineshkumar B, Krishnakumar K, Bhatt AR, Paul D, Cherian J John A and Suresh S: Single-walled and multi-walled carbon nanotubes based drug delivery system: Cancer therapy: A review. Indian J Cancer 52: 262-274, 2015.

10. Al Faraj A, Shaik AP and Shaik AS: Magnetic single-walled carbon nanotubes as efficient drug delivery nanocarriers in breast cancer murine model: Noninvasive monitoring using diffusion-weighted magnetic resonance imaging as sensitive imaging biomarker. Int J Nanomedicine 10: 157-168, 2014.

11. Liu J, Wang C, Wang X, Wang X, Cheng L, Li Y and Liu Z: Mesoporous silica coated single-walled carbon nanotubes as a multifunctional light-responsive platform for cancer combination therapy. Adv Funct Mater 25: 384-392, 2015.

12. Zhang W, He J, Liu Z, Ni P and Zhu X: Biocompatible and pH-responsive triblock copolymer mPEG-b-PCL-b-PDMAEMA: Synthesis, self-assembly, and application. J Polymer Sci Part A Polymer Chem 48: 1079-1091, 2010.

13. Das M, Bandyopadhyay D, Singh R, Harde H, Kumar S and Jain SL: Orthogonal bio-functionalization of magnetic nano-particles via 'clickable' poly (ethylene glycol) silanes: A 'universal ligand' strategy to design stealth and target-specific nano-carriers. J Mater Chem 22: 24652-24667, 2012.
14. Moghimi SM, Hunter AC and Murray JC: Long-circulating and target-specific nanoparticles: Theory to practice. Pharmacol Rev 53: 283-318, 2001

15. Schiff R, Massarweh S, Shou J and Osborne CK: Breast cancer endocrine resistance: How growth factor signaling and estrogen receptor coregulators modulate response. Clin Cancer Res 9: 447S-454S, 2003.

16. Kurebayashi J, Otsuki T, Kunisue H, Tanaka K, Yamamoto S and Sonoo H: Expression levels of estrogen receptor-alpha, estrogen receptor-beta, coactivators, and corepressors in breast cancer. Clin Cancer Res 6: 512-518, 2000.

17. Horwitz KB and McGuire WL: Estrogen control of progesterone receptor in human breast cancer: Correlation with nuclear processing of estrogen receptor. J Biol Chem 253: 2223-2228, 1978.

18. Rai S, Paliwal R, Vaidya B, Gupta PN, Mahor S, Khatri K, Goyal AK, Rawat A and Vyas SP: Estrogen(s) and analogs as a non-immunogenic endogenous ligand in targeted drug/DNA delivery. Curr Med Chem 14: 2095-2109, 2007.

19. Keely NO and Meegan MJ: Targeting tumors using estrogen receptor ligand conjugates. Curr Cancer Drug Targets 9: 370-380, 2009.

20. Dao KL and Hanson RN: Targeting the estrogen receptor using steroid-therapeutic drug conjugates (hybrids). Bioconjug Chem 23: 2139-2158, 2012

21. Datir SR, Das M, Singh RP and Jain S: Hyaluronate tethered, 'smart' multiwalled carbon nanotubes for tumor-targeted delivery of doxorubicin. Bioconjug Chem 23: 2201-2213, 2012.

22. Yu SP, Su XD, Du JL, Wang JL, Gao YD, Zhang L, Chen L, Yang YZ and Liu XG: The cytotoxicity of water soluble carbon nanotubes on human embryonic kidney and liver cancer cells. New Carbon Materials 33: 35-46, 2018.

23. Carlson JC, Stefan MI, Parnis JM and Metcalfe CD: Direct UV photolysis of selected pharmaceuticals, personal care products and endocrine disruptors in aqueous solution. Water Res 84: 350-361, 2015.

24. Salvador-Morales C, Townsend P, Flahaut E, Vénien-Bryand C, Vlandase A, Greena MLH and Sim RB: Binding of pulmonary surfactant proteins to carbon nanotubes; potential for damage to lung immune defense mechanisms. Carbon 45: 607-617, 2007.

25. Wang DP, Li SR, Zhang M, Li ST, Zhang YP, Geng ZX and Li RF: Determination of Hematological and Biochemical Parameters in Three Stocks of Mice. Shiyandongwu Kexue Yu Guanli 17: 24-28, 2000 (In Chinese).

(i) This work is licensed under a Creative Common Attribution 4.0 International (CC BY 4.0) License. 